\title{
Does binocular disparity facilitate the detection of transparent motion?
}

\author{
Paul B Hibbard, Mark F Bradshaw \\ Department of Psychology, University of Surrey, Guildford, Surrey GU2 5XH, UK; \\ e-mail: P.Hibbard@surrey.ac.uk \\ Received 16 February 1998, in revised form 8 May 1998
}

\begin{abstract}
Recent physiological studies have established that cortical cells that are tuned for the direction of motion may also exhibit tuning for binocular disparity. This tuning does not appear to provide any advantage in discriminating the direction of global motion in random-dot kinematograms. Here we investigated the possibility that this tuning may be important in the perception of transparent motion. Random-dot kinematograms were presented which contained coherent motion in a single direction or in two opposing directions. A greater proportion of signal dots was required for the detection of transparent motion than of motion in a single direction. This difference vanished when the two opposite directions of motion were presented with different disparities. These results suggest that the direction of global motion can be computed separately for surfaces which are clearly segregated in depth.
\end{abstract}

\section{Introduction}

Physiological evidence has shown that binocular disparity and motion information are combined during visual processing. Neurons in cortical areas V1 (Poggio and Talbot 1981), V5/MT (Maunsell and van Essen 1983; Bradley et al 1995), and MST (Komatsu et al 1988; Roy et al 1992) have been found which exhibit tuning for both the direction of motion and binocular disparity. An interplay between motion and stereopsis has also been established psychophysically (eg Rogers and Graham 1984; Nawrot and Blake 1991; Bradshaw and Rogers 1996). This joint tuning, especially noted in area MT, suggests that binocular disparity may play a rôle in motion perception. One function of area MT would appear to be the integration of motion signals, leading to the representation of the global direction of motion within an image region. For example, MT cells in the macaque monkey have been shown to respond selectively to the direction of coherent motion in plaid stimuli (Movshon et al 1985; Rodman and Albright 1989) and to the global direction of motion in random-dot kinematograms (RDKs) in which a coherent motion is embedded in motion noise (Newsome and Pare 1988). It might be expected therefore that the detectability of the global direction of motion in RDKs would be influenced by binocular disparity. However, Hibbard et al (1998) found that when a coherent motion was carried by dots that were intermingled in depth with other dots that were moving in random directions, the coherence required to detect the direction of motion was similar to that required when all signal and noise dots were presented with zero disparity. Manipulating the spread of signal and noise dots through depth (defined by disparity) did not lead to an improvement in performance, even when this resulted in the majority of noise dots being presented with a different disparity from that of the signal dots. This suggests that the perception of global motion is not influenced by binocular disparity, despite the linkage between these cues exhibited by many cells in area MT.

The potential functional significance of the joint tuning for motion direction and binocular disparity was therefore explored further here by investigating the rôle of disparity in the perception of transparent motions in opposite directions. This was motivated by the results of a number of recent studies which suggest that motion transparency is processed in area MT, and that neurons in this area show clear disparity 
tuning in their responses to motion transparency. Qian et al (1994) studied the perception of transparent motion in random-dot displays in which pairs of dots moved over each other horizontally in opposite directions. Transparency was not readily perceived in these displays. If, however, the two dots in each pair were separated vertically, transparent motion was observed. In a related physiological study, Qian and Andersen (1994) reported that the majority of directionally selective V1 cells in the macaque responded equally well to transparent motion in which dots moving in opposite directions were either paired or unpaired. In contrast, the response elicited from the majority of MT cells by transparent motion in which dots were spatially paired was much reduced relative to that elicited by transparent motion in unpaired stimuli. Qian and Andersen suggested therefore that MT represents the cortical locus for the perception of transparent motion, although they also found that the magnitude of the responses to transparent motion was much less than that for motion in the preferred direction. Bradley et al (1995) also found that the responses of MT cells to motion in their preferred direction were much reduced by the presence of motion in the opposite direction. This reduced level of activation, however, was less pronounced when the two directions of motion were presented with different binocular disparities. They suggested that transparent stimuli induce mutual suppression between mechanisms tuned to different directions of motion, and that this suppression is tuned for binocular disparity.

These results drawn from the physiological domain clearly predict that transparent motion would be more readily detectable when different directions of motion are presented with different disparities. Qian et al (1994) demonstrated that the appearance of transparency in RDKs could be enhanced by segregating dots moving in different directions onto different disparity planes. In these stimuli, however, binocular disparity in itself acts as a strong cue to transparency (Akerstrom and Todd 1988), so it is not clear whether the increased salience of transparency achieved was necessarily related to the motion present in the stimuli. Here, we investigate whether binocular disparity can affect the perception of transparent motion in RDKs using a task which could only be performed on the basis of motion cues.

\section{Experiment 1}

Previous physiological (Snowden et al 1991; Qian and Andersen 1994) and psychophysical (Lappin and Kottas 1981; Mather and Moulden 1983; Snowden 1989; Lindsey and Todd 1995) studies have demonstrated that transparent motion is less readily perceived than is motion in a single direction. In the first experiment, we measured the coherence required to detect the two types of motion, for stimuli in which all elements were presented with zero disparity. We used RDKs in which a target motion was carried by a proportion of the dots (the signal dots), while the remainder (the noise dots) moved in random directions. In the terminology of Snowden et al (1991), the target motion was either single-surface motion, in which all signal dots moved in the same direction, or transparent motion, in which half the signal dots moved in one direction, and the other half in the opposite direction.

\subsection{Methods}

2.1.1 Procedure. In separate blocks of trials, we measured the coherence that was required for observers to detect single-surface motion and transparent motion. In a $2 \mathrm{AFC}$ procedure, two RDKs were presented on each trial, separated by an interstimulus interval of $500 \mathrm{~ms}$. On every trial, one interval was designated as the 'target-present' interval (which contained signal and noise), and the other as the 'target-absent' interval (which contained only noise). The observers' task was to decide which interval contained the target. Between trials, the coherence of the target-present RDKs (the proportion of the dots that were assigned as signal dots) was varied. Coherence thresholds were 
measured by the method of constant stimuli. Psychometric functions were obtained on the basis of 40 observations at each of 6 coherence levels, which were selected on the basis of pilot studies. Frequency-of-seeing plots were generated from each data set and the best-fitting cumulative Gaussian curve was determined by the probit technique (Finney 1971). Thresholds represent the $75 \%$ correct point of the fitted function; error bars represent the standard error of the parameter estimates, which were also determined by the probit procedure.

2.1.2 Stimuli. Each RDK consisted of two frames, each presented for $150 \mathrm{~ms}$, with no interframe interval. The RDKs contained 200 dots, which were presented in a $4 \mathrm{deg} \times 4 \mathrm{deg}$ square window. Stimuli were surrounded by an $8 \mathrm{deg} \times 8 \mathrm{deg}$ square of static random dots, with a density of 12.5 dots $\mathrm{deg}^{-2}$. Individual dots were formed from Gaussian blobs with a spatial standard deviation of $2.5 \mathrm{~min}$ of arc, positioned with subpixel accuracy. The Gaussian blobs had a maximum luminance of $73.0 \mathrm{~cd} \mathrm{~m}^{-2}$. The background luminance of the screens was $0.4 \mathrm{~cd} \mathrm{~m}^{-2}$. Dots were repositioned between frames in the following manner.

Single-surface motion. In the target-present RDKs, a proportion of the dots was randomly assigned as signal dots. All signal dots were moved $8 \mathrm{~min}$ of arc in the same horizontal direction (left or right) between frames. The remaining dots (the noise dots) were each replaced $8 \mathrm{~min}$ of arc from their original position in a random direction. For each noise dot, this direction was chosen from a rectangular distribution, covering the full $360^{\circ}$ of possible directions. A single-surface motion stimulus is illustrated in figure 1a.
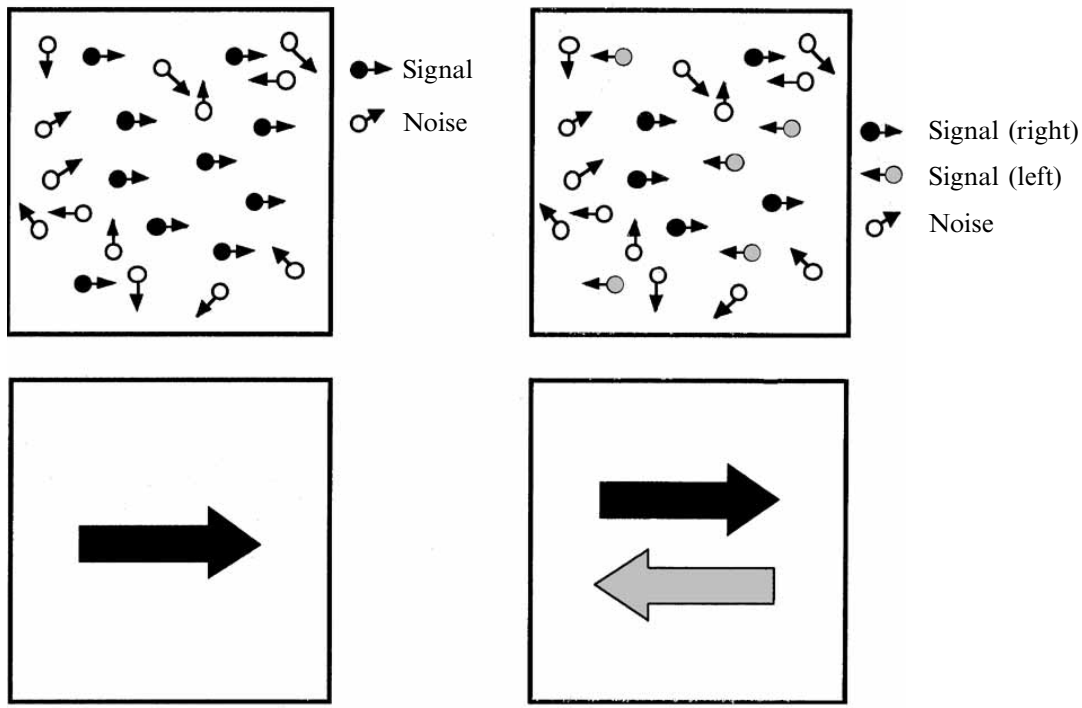

(a)

(b)

Figure 1. Stimuli used in the experiment. (a) A single-surface-motion stimulus. Signal dots (shown here in black) were all moved in the same direction (here to the right) between frames. Noise dots (shown here in white) were moved in random directions. (b) A transparent-motion stimulus. Half of the signal dots (shown here in grey) were moved to the left between frames, and the remainder (shown in black) were moved to the right. Noise dots (again shown in white) were moved in random directions.

In the target-absent RDKs, all dots were assigned as noise dots, and were replaced $8 \mathrm{~min}$ of arc from their original position between frames, in a random direction. Again, the direction for each dot was chosen from a rectangular distribution, covering the full $360^{\circ}$ of possible directions. 
Transparent motion. In the target-present RDKs, a proportion of the dots was randomly assigned as signal dots. Of these, half were assigned as leftward-moving signal dots, and were moved $8 \mathrm{~min}$ of arc to the left between frames. The remaining half were assigned as rightward-moving signal dots, and were moved $8 \mathrm{~min}$ of arc to the right between frames. The remaining dots (the noise dots) were each replaced $8 \mathrm{~min}$ of arc from their original position, in a random direction. For each noise dot, this direction was again chosen from a rectangular distribution, covering the full $360^{\circ}$ of possible directions. A transparent motion stimulus is illustrated in figure 1b. Target-absent RDKs were identical to those used in the single-surface motion condition.

2.1.3 Observers. The two authors served as observers. Both observers had good stereopsis, and had normal or corrected-to-normal vision.

2.1.4 Apparatus. Stimuli were presented on two Apple 12 inch monochrome monitors, driven by a Macintosh 7500 and arranged in a standard Wheatstone stereoscope configuration. The monitors were viewed through two first-surface mirrors set at $\pm 45^{\circ}$ to the median plane. The viewing distance was $114 \mathrm{~cm}$, at which each pixel subtended $1 \mathrm{~min}$ of arc. The experiments were carried out in a dimly lit room.

\subsection{Results and discussion}

Coherence thresholds for detecting single-surface and transparent motion are plotted in figure $2 \mathrm{a}$. Fewer signal dots were required to detect single-surface motion than to detect transparent motion. Coherent thresholds for detecting transparent motion were on average 1.84 times those for detecting single-surface motion.

The coherence thresholds plotted in figure $2 \mathrm{a}$ represent the percentage of the total number of dots in a stimulus that were assigned as signal dots. While this accurately describes the creation of the stimuli, it does not necessarily reflect the manner in which they are processed by the visual system. In single-surface motion all signal dots moved in the same direction, whereas in transparent motion half moved in one direction and half in the opposite direction. It is possible that motion coherence thresholds were determined simply by the number of signal dots moving in a particular direction, relative to the total number of dots in the stimulus. If this were the case, then one would predict that twice as many signal dots would be required to detect transparent motion as are required to detect single-surface motion, since in the former case the signal dots are shared equally between two directions of motion. In figure $2 b$, the data are replotted to show the percentage of the total number of dots that are moving to the left, or equivalently the percentage of the total moving to the right. When plotted this way, thresholds for transparent and coherent motion are very similar. These results
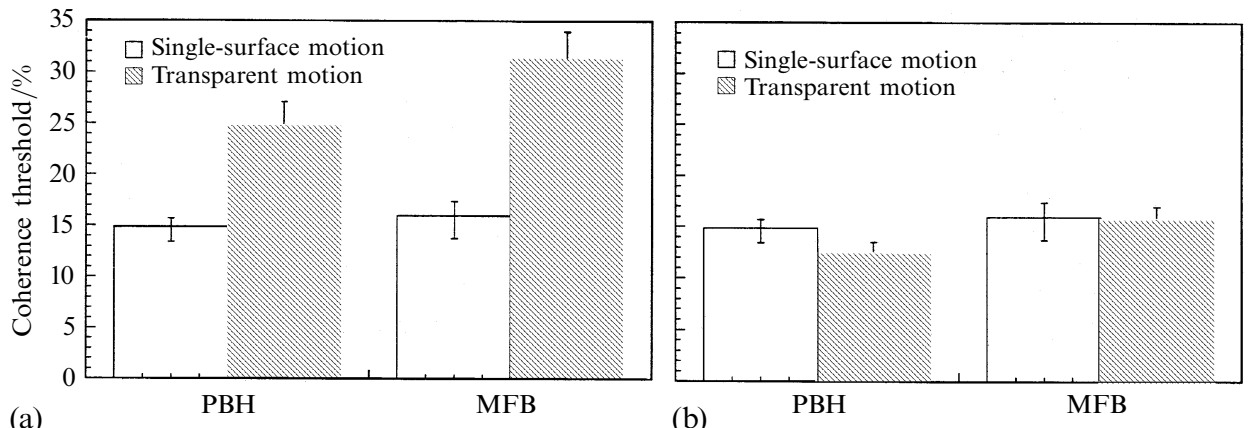

(a)

(b)

Figure 2. Thresholds for the detection of motion for single-surface and transparent motion. Results are plotted (a) as the percentage of the total number of dots that were assigned as signal dots and (b) as the percentage of the total number of dots that were assigned as signal dots moving in a single direction. Error bars represent \pm 1 standard error. 
imply that the detection of transparent motion relies on the independent detection of the individual component motions. Further, the close similarity of the thresholds presented in figure $2 \mathrm{~b}$ suggests that the subset of dots moved to the right, as well as the subset of dots moved in random directions, acted to inhibit detection of the motion to the left (and vice versa). These results are consistent with previous reports that transparent motion is less detectable than single-surface motion (Lappin and Kottas 1981; Mather and Moulden 1983; Snowden 1989; Lindsey and Todd 1995). The results suggest that transparent motion is detected by mechanisms selective for the individual directions of motion comprising the transparency, rather than by a mechanism specialised for the detection of transparent motion.

\section{Experiment 2}

Experiment 1 established baseline thresholds for single-surface and transparent motion when the stimuli were presented with zero disparity. Experiment 2 was designed to determine whether thresholds for the detection of transparent motion were affected by separating the two directions of motion in depth by using binocular disparity. If motion is detected by mechanisms selective for both direction and binocular disparity, then an improvement in performance might be expected for stimuli containing dots presented on two planes separated in depth by binocular disparity. Moreover, physiological evidence suggests that transparent motion is detected by specialised mechanisms tuned to different directions of motion presented at different disparities (Roy et al 1992). It is possible therefore that thresholds for transparent motion may be lower than that predicted if motion is detected by mechanisms tuned for binocular disparity and for a single direction of motion.

\subsection{Method}

The design, procedure, and observers were the same as described in experiment 1.

3.1.1 Stimuli. All RDKs comprised two frames, each containing 200 dots. Target-present RDKs contained transparent motion, as in the previous experiment. On half of the trials, leftward-moving signal dots were presented with a crossed disparity, and rightward-moving signal dots were presented with an uncrossed disparity of the same magnitude. On the remaining half of the trials, the pattern was reversed, ie leftwardmoving dots were presented with uncrossed disparity, and rightward-moving dots with crossed disparity. Trials of each type were intermingled randomly. On all trials, half of the noise dots were presented on each of these two disparity planes. Crossed and uncrossed disparities of $0.5,1,2,4$, and $8 \mathrm{~min}$ of arc were used, so that the separation between the two planes was 1, 2, 4, 8, or 16 min of arc. For observer PBH a threshold was also measured for dots presented with crossed and uncrossed disparities of $1.5 \mathrm{~min}$ of arc.

As before, in the target-absent RDKs all dots were assigned as noise dots, and moved $8 \mathrm{~min}$ of arc in a random direction between frames. Half of the noise dots in this experiment were presented with crossed disparity (with the same magnitude as that used in the target-present RDK for that trial), and half were presented with uncrossed disparity of the same magnitude. Therefore, to distinguish the stimuli it was necessary to detect the motion signal, as the disparity was identical in both intervals.

\subsection{Results and discussion}

Coherence thresholds are plotted against the disparity separation between the two planes in figure 3. Results are plotted separately for the two observers. As the separation between the planes increased, thresholds decreased, reaching a minimum for a separation of $4 \mathrm{~min}$ of arc for $\mathrm{PBH}$, and $2 \mathrm{~min}$ of arc for MFB. Thresholds increased for disparity separations greater than these values. For separations of $16 \mathrm{~min}$ of arc, thresholds for both observers were equal to those obtained when both directions of 

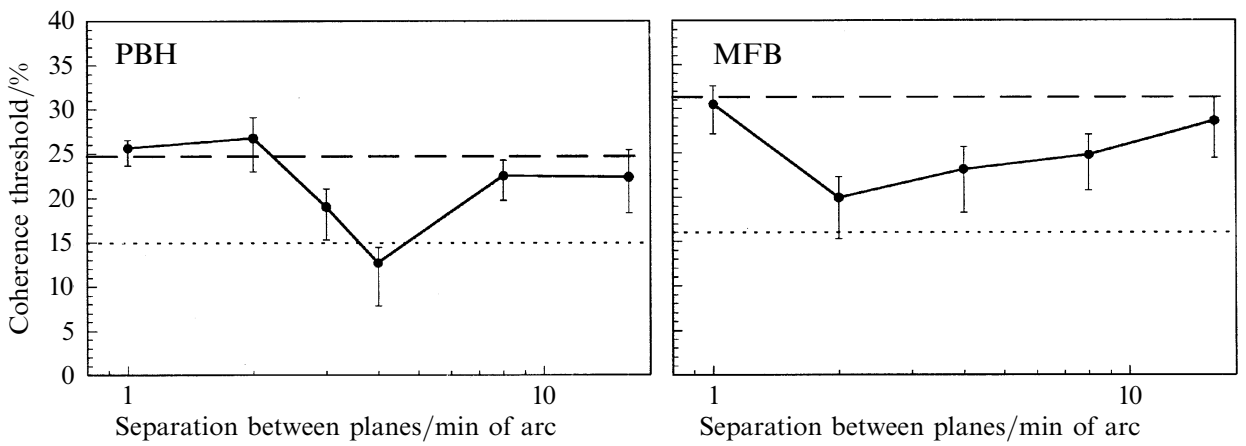

Figure 3. Thresholds for the detection of transparent motion plotted against the separation in depth between the two directions of motion. The dotted horizontal line on each graph shows the threshold for detecting single-surface motion, the dashed horizontal line the threshold for detecting transparent motion when all dots were presented with zero disparity (both replotted from figure 2a). Error bars represent \pm 1 standard error.

motion were presented with zero disparity. For the largest disparity separation diplopia was evident and the dots did not appear to lie on two separate planes in depth. In this case, motion processing presumably relies on monocularly driven mechanisms. Similar effects of disparity on the detection of motion have been obtained in other studies (eg McKee et al 1997; Hibbard et al 1998). Probit analysis was used to compare the lowest threshold obtained for each observer (at a separation of $4 \mathrm{~min}$ of arc for $\mathrm{PBH}$, and 2 min of arc for MFB) with the thresholds obtained in the first experiment (Finney 1971). For both observers, the difference in thresholds was significant $(p<0.05)$.

The minimum coherence thresholds reached for transparent motion are similar to those obtained for the detection of single-surface motion. The dotted horizontal lines on the graphs in figure 3 show coherence thresholds for the detection of single-surface motion that were measured for each observer in the previous experiment. Fewer signal dots were required on each plane to detect the signal motion than were required for the detection of either single-surface or transparent motion when all dots were presented with zero disparity. These results suggest that global motion was analysed independently on the two planes of dots and little interaction (of the oppositely moving signals) took place between them. Each signal motion was presented on a plane containing only half the total number of dots present in the stimulus. Since motion coherence thresholds are typically related to the number of signal dots in a stimulus relative to the total number of dots (eg Scase et al 1996), these thresholds are what would be expected if coherence was determined on each of the planes separately. Our results therefore are consistent with the notion that the processing of motion can take place within disparity-tuned channels, as coherence was determined for each disparitydefined plane independently of the other. No additional improvement in performance was observed, beyond that expected by considering the motion coherence on each of the two planes independently. It is possible that this is because observers were able to perform the task on the basis of the motion signal on each of the two planes, and were not forced or required to utilise information from both planes simultaneously.

\section{General discussion}

Coherence thresholds for the detection of transparent motion were found to be greater than coherence thresholds for the detection of motion in a single direction in experiment 1 . However, when coherence thresholds were reassessed in terms of the proportion of dots moving in a single direction, thresholds were comparable for transparent and single-surface motion. These results suggest that transparent motion in these stimuli was detected by distinct mechanisms tuned to the two directions of motion present, 
and are consistent with previous reports that transparent motion is less readily detected than motion in a single direction (Lappin and Kottas 1981; Mather and Moulden 1983; Snowden 1989; Lindsey and Todd 1995). In experiment 2, coherence thresholds for the detection of transparent motion decreased when the stimulus was presented on two planes, separated in depth by using binocular disparity.

The results reported here may be related to other recent investigations into the effects of binocular disparity on the detectability of global motion. For example, Hibbard et al (1998) found no improvement in performance when signal dots were presented on a single plane, embedded within a cloud of noise dots, compared to when signal and noise dots were all presented with the same disparity. This suggested that global motion is not disparity tuned. However, Hibbard et al showed that if the signal dots are presented with a different disparity to all of the noise dots, coherence thresholds are considerably lower than if all the signal and noise dots are presented with the same disparity. These authors argued that disparity influenced global motion perception only in the situation where it could be used to clearly segregate the motions into distinct planes at different depths, and concluded that in these circumstances an attentional mechanism may influence the results. Similarly, Snowden and Rossiter (1999) found that the perception of coherent motion was unaffected by the presence of noise dots on a plane, defined by binocular disparity, distinct to that containing the signal in the same stimulus. The results of the second experiment of the current study are similar to those reported by Snowden and Rossiter. The disparity used by Snowden and Rossiter was approximately $6 \mathrm{~min}$ of arc, near to the peak in the tuning function found in the current experiment.

Therefore, taken together it appears that disparity can affect the perception of global motion only if it provides a clear surface segmentation cue. This clear segmentation appears necessary in order for processing to be selectively guided to the output of a particular disparity-tuned motion processing mechanism, and may be related to the rôle of attention in modulating the responses of MT and MST cells that has been reported physiologically (Treue and Maunsell 1996). In the stimuli used by Hibbard et al the planes containing the signal dots were embedded within the volume containing the noise dots and were not therefore perceptually salient. Snowden and Rossiter similarly concluded that the segregation of a stimulus into distinct surfaces is required in order to selectively ignore a subset of the dots.

The rôle of binocular disparity in the perception of transparent motion has been addressed in several other studies. Von Grünau et al (1993) studied moving plaid patterns in which component gratings were presented either with the same or with a different disparity. Transparent motion was more frequently observed when components were presented with different disparities than when components had the same disparity. Verstraten et al (1994) studied motion aftereffects (MAEs) elicited by transparent motion. While no MAE was elicited by a stimulus containing motions in opposite directions which were presented with the same disparity, disparity-contingent MAEs were observed when the two directions were presented with different disparities. Qian et al (1994) found that transparency was more readily observed in paired random-dot patterns if pairs of dots were presented with different disparities. All of the above results support the view that motion processing can occur relatively independently at different disparities. One example where this has not been found is in direction repulsion. Hiris and Blake (1996) reported that repulsion between different directions of motion occurred regardless of whether the two motion signals were presented with the same or different disparities. They concluded that the process responsible for direction repulsion occurs prior to selectivity for binocular disparity. 
Other examples of interactions between stereo and motion are common in the literature. For example, Anstis and Harris (1974) demonstrated that motion aftereffects are contingent on binocular disparity. Depth aftereffects that were contingent on the direction of motion were also found. Other interactions have been observed in the kinetic depth effect (Nawrot and Blake 1991), in shape-from-motion and stereopsis (eg Johnston et al 1994; Bradshaw and Rogers 1996), and in the resolution of stereoscopic correspondence (Bradshaw and Cumming 1997). These results are consistent with physiological reports that motion direction and binocular disparity information are integrated in processing.

It is interesting to speculate on the site where the interactions between motion and stereopsis occur. As discussed earlier, motion processing is often characterised as a two-stage process. The first stage is believed to be performed by motion detectors with small receptive fields, which are tuned to the orientation, scale, and velocity of image features lying within particular retinal regions. In the second stage, these local measurements are combined to form a representation of the global motion within larger areas (see eg Braddick 1993). These local and global processes have been associated with processing in cortical areas V1 and MT, respectively. As both areas contain neurons tuned for both direction of motion and binocular disparity, it is possible that the interactions found psychophysically could occur either at the local or the global level. The RDK stimuli used in the current study, and by Hibbard et al (1998) and Snowden and Rossiter (1999) were designed so that motion detection and discrimination cannot be based on the motions of individual dots. Since there is no information available locally to determine which dots are signal and which are noise, the global direction of motion can only be determined by considering the motion of the dots as a whole. If the signal dots are labelled by giving them a different disparity (Hibbard et al 1998; Snowden and Rossiter, submitted) or colour (Edwards and Badcock 1996; Croner and Albright 1997) to the noise dots, coherence thresholds are considerably lower, as the task can be performed by considering only the motion of dots with a particular disparity or colour. This task may in principle be performed at the level of local motion detection, which is considered to occur in V1. In contrast, in those conditions in which noise dots were also presented with the disparity of the signal dots, as in the current study, global motion mechanisms must be involved. These results therefore provide support for the notion that global motion processing can occur independently for stimuli presented with different disparities.

\section{References}

Akerstrom R A, Todd T J T, 1988 "The perception of stereoscopic transparency" Perception \& Psychophysics $44421-432$

Anstis S M, Harris J P, 1974 "Movement aftereffects contingent on binocular disparity" Perception $3153-168$

Braddick O J, 1993 "Segmentation versus integration in visual motion processing" Trends in Neuroscience $16263-268$

Bradley D C, Qian N, Andersen R A, 1995 "Integration of motion and stereopsis in middle temporal cortical area of macaques" Nature (London) 373 609-611

Bradshaw M F, Rogers B J, 1996 "The interaction of binocular disparity and motion parallax in the computation of depth" Vision Research 363457 -3468

Bradshaw M F, Cumming B G, 1997 "The direction of motion facilitates binocular stereopsis" Proceedings of the Royal Society of London, Series B 2641421 - 1427

Croner L J, Albright T D, 1997 "Image segmentation enhances discrimination of motion in visual noise" Vision Research $371415-1427$

Edwards M, Badcock D R, 1996 "Global-motion perception: interaction of chromatic and luminance signals" Vision Research $362423-2431$

Finney D J, 1971 Probit Analysis (Cambridge: Cambridge University Press)

Grünau M von, Dabé S, Kwas M, 1993 "The effect of disparity on motion coherence" Spatial Vision $7227-241$ 
Hibbard P B, Bradshaw M F, DeBruyn B, 1998 "Is global motion tuned for binocular disparity?" Vision Research (in press)

Hiris E, Blake R, 1996 "Direction repulsion in motion transparency" Visual Neuroscience 13 $187-197$

Johnston E B, Cumming B G, Landy M S, 1994 "Integration of stereopsis and motion shape cues" Vision Research $342259-2275$

Komatsu H, Roy J P, Wurtz R H, 1988 "Binocular disparity sensitivity of cells in area MST of the monkey" Society for Neuroscience Abstracts 14202

Lappin J S, Kottas B L, 1981 "The perceptual coherence of moving visual patterns" Acta Psychologica $48163-174$

Lindsey D T, Todd J T, 1995 "Detection of motion in transparent motion displays" Investigative Ophthalmology \& Visual Science 36(4) S1048

McKee S P, Watamaniuk S N J, Harris J M, Smallman H S, Taylor D G, 1997 "Is stereopsis effective in breaking camouflage for moving targets?" Vision Research 37 2047-2055

Mather G, Moulden B, 1983 "Thresholds for movement direction: two directions are less detectable than one" Quarterly Journal of Experimental Psychology 35 513-518

Maunsell J H R, Van Essen D C, 1983 "Functional properties of neurons in the middle temporal area of the macaque monkey, II: Binocular interactions and sensitivity to binocular disparity" Journal of Neurophysiology $491148-1167$

Movshon J A, Adelson E A, Gizzi M, Newsome W T, 1985 "The analysis of moving visual patterns", in Study Group on Pattern Recognition Mechanisms Eds C Chagis, R Gattass, C G Gross (Vatican City: Pontifica Academia Scientiarum) pp 117 - 151

Nawrot M, Blake R, 1991 "The interplay between stereopsis and structure from motion" Perception \& Psychophysics $49230-244$

Newsome W T, Paré E B, 1988 "A selective impairment of motion perception following lesions of the middle temporal visual area (MT)" Journal of Neuroscience $82201-2211$

Poggio G F, Talbot W H, 1981 "Mechanisms of static and dynamic stereopsis in foveal cortex of the rhesus monkey" Journal of Physiology (London) $315469-492$

Qian N, Andersen R A, 1994 "Transparent motion perception as detection of unbalanced motion signals. II. Physiology" Journal of Neuroscience $147367-7380$

Qian N, Andersen R A, Adelson E H, 1994 "Transparent motion perception as detection of unbalanced motion signals. I. Psychophysics" Journal of Neuroscience $147357-7366$

Rodman H R, Albright T D, 1989 "Single-unit analysis of pattern motion selective properties in the middle temporal visual area (MT)" Experimental Brain Research 75 53-64

Rogers B J, Graham M E, 1984 "Aftereffects from motion parallax and stereoscopic depth: similarities and interactions", in Sensory Experience, Adaptation and Perception: Festschrift Ivo Kohler Eds L Spillmann, B R Wooten (Hillsdale, NJ: Lawrence Erlbaum Associates) pp 603-619

Roy J P, Komatsu H, Wurtz R H, 1992 "Disparity sensitivity of neurons in monkey extrastriate area MST" Journal of Neuroscience $122478-2492$

Scase M O, Braddick O J, Raymond J E, 1996 "What is noise for the motion system?" Vision Research $362579-2586$

Snowden R J, 1989 "Motions in orthogonal directions are mutually suppressive" Journal of the Optical Society of America A $71096-1101$

Snowden R J, Rossiter M C, 1999 "Stereoscopic depth cues can segment motion information" Perception $28193-201$

Snowden R J, Treue S, Erickson R G, Andersen R A, 1991 "The response of area MT and V1 neurons to transparent motion noise" Journal of Neuroscience $112768-2785$

Treue S, Maunsell J H R, 1996 "Attentional modulation of visual motion processing in cortical areas MT and MST" Nature (London) $382539-541$

Verstraten F A J, Verlinde R, Fredricksen R E, Grind W A van de, 1994 "A transparent motion aftereffect contingent on binocular disparity" Perception 231181 - 1188 
